\title{
Methods of Stochastic Analysis of Complex Regimes in the 3D Hindmarsh-Rose Neuron Model
}

\author{
Irina Bashkirtseva, Lev Ryashko* and Evdokia Slepukhina \\ Institute of Mathematics and Computer Science \\ Ural Federal University \\ Lenina 51, Ekaterinburg 620083, Russia \\ *Lev.Ryashko@urfu.ru
}

Received 24 May 2016

Accepted 15 November 2017

Published 22 January 2018

Communicated by Benjamin Lindner

\begin{abstract}
A problem of the stochastic nonlinear analysis of neuronal activity is studied by the example of the Hindmarsh-Rose (HR) model. For the parametric region of tonic spiking oscillations, it is shown that random noise transforms the spiking dynamic regime into the bursting one. This stochastic phenomenon is specified by qualitative changes in distributions of random trajectories and interspike intervals (ISIs). For a quantitative analysis of the noise-induced bursting, we suggest a constructive semi-analytical approach based on the stochastic sensitivity function (SSF) technique and the method of confidence domains that allows us to describe geometrically a distribution of random states around the deterministic attractors. Using this approach, we develop a new algorithm for estimation of critical values for the noise intensity corresponding to the qualitative changes in stochastic dynamics. We show that the obtained estimations are in good agreement with the numerical results. An interplay between noise-induced bursting and transitions from order to chaos is discussed.
\end{abstract}

Keywords: Hindmarsh-Rose model; excitability; stochastic sensitivity; noise-induced transitions; stochastic generation of bursting oscillations; stochastic bifurcations; noise-induced chaotization.

\section{Introduction}

In recent years, new mathematical methods of nonlinear dynamics are widely applied to neuroscience. A number of interesting results on the dynamics of neural systems were obtained, and many types of neural behavior received adequate interpretation in terms of dynamical systems theory [1]. In a variety of studies of neurons, both experimental and theoretical, specific types of activity have been found, combining a quiescence and an excitability [1,2]. The excitability can be realized in the form of the sequence of sharp periodic spikes or as an alternation of quiescence and spiking, so-called bursting. 
For the mathematical description of the neural behavior, various nonlinear dynamic models were elaborated. Here, an important place belongs to the models of ionic transport through neuron membrane, based on Hodgkin-Huxley equations [3]. This four-dimensional model can perfectly fit the experimental data but it is quite complicated for the analytical investigation, so low-dimensional mathematical models that are amenable to study are widely used. Here, 2D-models of FitzHugh-Nagumo and Morris-Lecar attracted the attention of many researchers [4-7]. Due to the bifurcation analysis of these models, a significant progress in the understanding of possible internal mechanisms of neural dynamics has been achieved. The next step was connected with the transition to the three-dimensional Hindmarsh-Rose (HR) model [8]. This system is one of the simplest models representing bursting neural activity.

Of particular interest is the study of the response of neural systems to random disturbances. Different sources of noise influence the behavior of a single neuron [9]. The phenomenon of channel noise is associated to the stochastic manner of opening of ion channels across the cell membrane. The synaptic noise comes from the activity of other neurons via synapses, which mediate connections between neurons. The current noise is connected to fluctuations of the applied current. In nonlinear models, noise can cause various effects that have no analogues in deterministic case. A wide range of new phenomena such as stochastic resonance [10-12], noise-induced transitions [13], noise-induced chaos [14], noise-induced order [15, 16], stochastic bifurcations [17] were discovered. Noise can play a constructive role in nonlinear models of neurodynamics. It was shown that even small stochastic fluctuations can lead to a significant qualitative changes in properties of such systems [5, 18, 19].

Deterministic models in neurodynamics are fairly well studied, while the research of the noise-induced phenomena remains an important and a challenging problem. The main tool in the vast majority of studies of nonlinear stochastic systems is the direct numerical simulation of random trajectories. For the solution of stochastic differential equations, various numerical schemes (explicit and implicit) were developed [20-22]. In order to provide the probabilistic analysis of stochastic phenomena, statistics calculated numerically for sufficiently large samples of data are used. The disadvantage of this method is that it requires a lot of computing resources. For a detailed parametric analysis of stochastic phenomena, the development of analytical approaches is needed.

The Fokker-Planck (FP) equation [23] gives the most detailed probabilistic description of stochastic dynamics. However, a direct use of this equation is very difficult even for the simplest cases. Under these circumstances, various asymptotics and approximations are developed. For the approximation of FP solutions, a well-known quasipotential method $[24,25]$ and a stochastic sensitivity function (SSF) technique [26, 27] can be applied.

The present paper is focused on the study of the noise-induced phenomena in the HR model of neuron activity. The deterministic HR model has been studied by many authors. The detailed bifurcation analysis of the system in different parametrical 
regions can be found in [28-33]. A particular attention of these studies has been devoted to the transition from the tonic spiking regime to the bursting one. As for the stochastic case, the effects of noise on the system with periodic external impulse, such as stochastic resonance [34-37] and coherence resonance [38, 39] were investigated. The effects of noise on the fast subsystem of the HR model were studied in [40].

This paper is organized as following. In Sec. 2, we discuss the basic properties and the bifurcations of the deterministic HR model. In Sec. 3, the effect of random disturbances on the system in the region of tonic spiking oscillations is studied. We show that under noise the spiking dynamic regime can transform to the bursting one. This phenomenon is confirmed by changes of distributions of random trajectories in the phase space and interspike intervals (ISIs). In Sec. 4, for a quantitative analysis of the noise-induced phenomena in the HR model, we suggest a constructive semi-analytical approach based on the SSF technique and the method of confidence domains. The algorithm of estimation of critical values for noise intensity corresponding to the noise-induced bursting is given. Dynamics of stochastic flows in HR model is studied in Sec. 5 in terms of Lyapunov exponents. Here, a relationship between phenomena of the noise-induced bursting and transitions from order to chaos is discussed.

\section{Deterministic System}

Consider the 3D HR model [8]:

$$
\begin{aligned}
& \dot{x}=y-x^{3}+3 x^{2}+I-z \\
& \dot{y}=1-5 x^{2}-y \\
& \dot{z}=r\left(s\left(x-x_{0}\right)-z\right),
\end{aligned}
$$

where $x$ is a membrane potential, variables $y, z$ describe fast and slow ionic currents, respectively, $I$ is a scalar (time-independent) parameter of an external current; $0<r \ll 1$ is a time scale parameter; $s, x_{0}$ are the parameters of the activation of the slow current. Bursting behavior requires a fast and a slow subsystem. Here, $x(t)$ and $y(t)$ form the fast subsystem of the model, whereas $z(t)$ is the slow subsystem. In this paper, we fix $r=0.002, s=4, x_{0}=-1.6$.

Consider the dynamics of the system (1) under variation of the parameter $I$. Figure 1(a) shows a bifurcation diagram of the deterministic system on the interval $I \in(1.0,4.0): z$-coordinates of equilibrium points, and $z$-coordinates of points of intersection of attractors with Poincare section plane $x=0$.

The system (1) has a single equilibrium which is stable for $I<I_{1} \approx 1.288$. It loses stability due to the subcritical Hopf bifurcation when the parameter $I$ passes from left to right through the point $I_{1}$.

For $I<I_{0} \approx 1.2677$, the stable equilibrium is a unique attractor of the system. As the parameter passes the point $I_{0}$, a stable limit cycle appears as a result of the saddle-node bifurcation. Such type of the cycle is termed "burst". For $I_{0}<I<I_{1}$, the system exhibits a coexistence of the stable limit cycle and the stable equilibrium. 


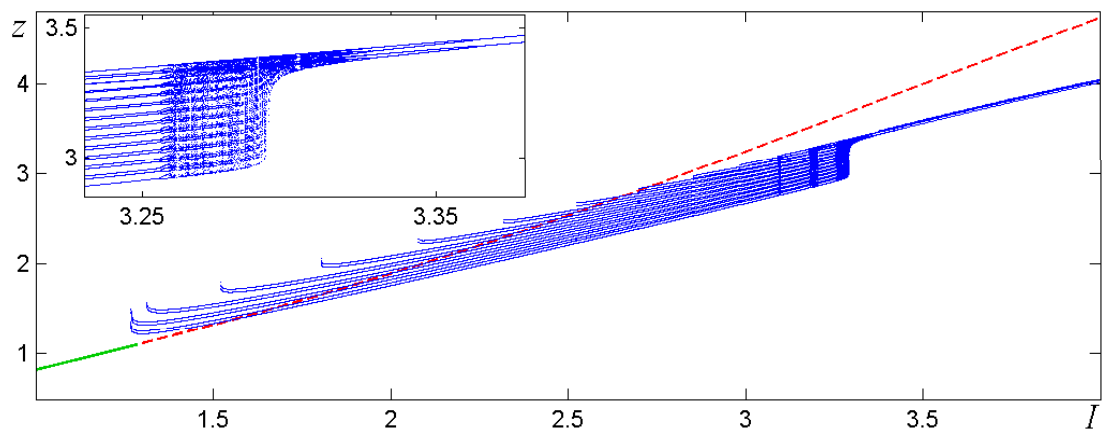

(a)
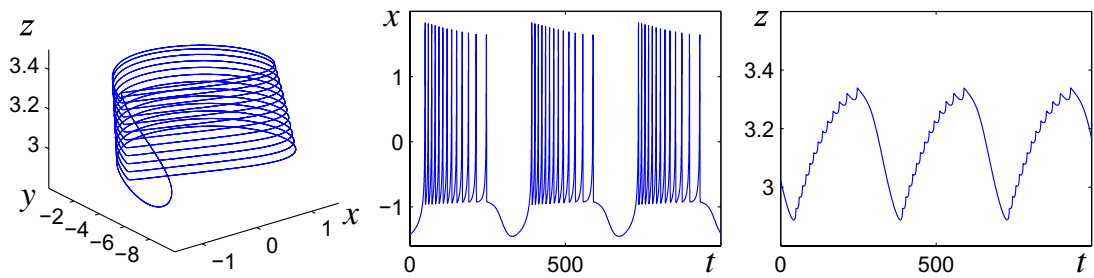

(b)
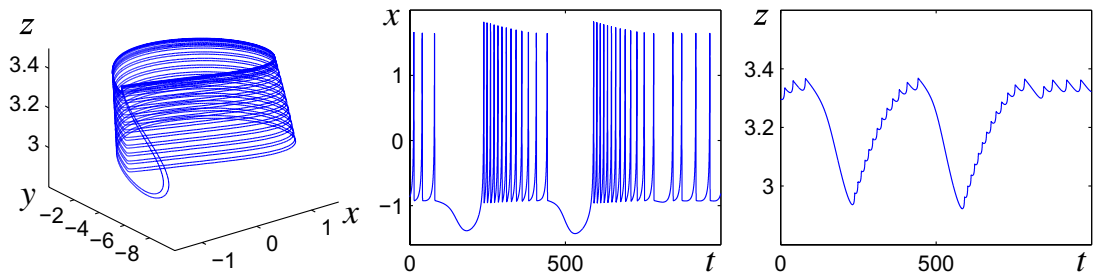

(c)
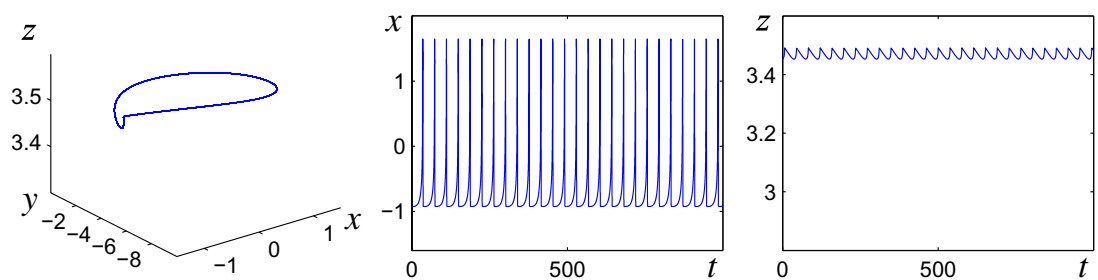

(d)

Fig. 1. Deterministic dynamics. (a) Bifurcation diagram: $z$-coordinates of stable (green solid) and unstable (red dashed) equilibrium points, and $z$-coordinates of points of intersection of limit cycles and chaotic attractors with Poincare section plane $x=0$ (blue solid). (b)-(d) Deterministic attractors and corresponding time series $x(t), z(t)$ for (b) $I=3.24$ (regular bursting), (c) $I=3.27$ (chaotic bursting), (d) $I=3.4$ (regular spiking).

Initially, near $I_{0}$, the limit cycle emerges with one spike per period. Then, with the increase of $I$, a sequence of period-adding bifurcations occurs: bursts with $2,3,4, \ldots$, 13 spikes appear. A transition from the burst with 12 spikes to the burst with 13 spikes is accompanied with the transition to chaos. The widest regions of the chaotic 
bursting are $3.177 \lesssim I \lesssim 3.206$ and $3.257 \lesssim I \lesssim 3.292$. For $I>I_{2} \approx 3.292$, a transition from the bursting chaos to the spiking chaos occurs. With further increase of $I$, the intermittency of chaotic and regular spiking is observed, then, at $I \approx 3.316$, the transition from the chaotic spiking regime to the regular one occurs. A sequence of backward period doubling bifurcations takes place: 4 -cycle transforms to 2 -cycle at $I \approx 3.328$, then a transition from 2 -cycle to 1 -cycle occurs at $I \approx 3.364$. Finally, the limit cycle transforms to the stable equilibrium due to the supercritical Hopf bifurcation at $I \approx 25.261$.

The classical mechanisms of neuronal bursting in mathematical models [41] are based on the separation of dynamics into the fast and slow components. For the HR model, at the value of $r=0.002$, used here, evolution of the variable $z$ is by orders of magnitude slower than dynamics of the variables $x$ and $y$. In the phase space of the HR model, there is a "slow surface", close to the plane $z=s\left(x-x_{0}\right)$ [28]. At the parameter values corresponding to deterministic bursting (in the current case, below $I=3.292$ ) creeping motion along the slow surface ("quiescent phase") alternates with rapid rotations far away from it ("the bursts"); transitions between these stages are caused by bifurcations in the "fast subsystem". At higher values of $I(I>3.292)$, the deterministic attractor (spiking state lies far away from the locus of those bifurcations and the bursts do not occur).

Figures 1(b)-1(d) shows the examples of typical regular bursting (Fig. 1(b)), chaotic bursting (Fig. 1(c)), and tonic spiking (Fig. 1(d)) attractors. In this paper, we focus on the parametric region of transition from the bursting regime to the spiking one: $I \in(3.0,4.0)$.

\section{Stochastic System}

Consider the stochastic HR model with an additive current noise:

$$
\begin{aligned}
& \dot{x}=y-x^{3}+3 x^{2}+I-z+\varepsilon \xi(t), \\
& \dot{y}=1-5 x^{2}-y \\
& \dot{z}=r\left(s\left(x-x_{0}\right)-z\right),
\end{aligned}
$$

where $\xi(t)$ is a white Gaussian noise with $\langle\xi(t)\rangle=0,\left\langle\xi(t) \xi^{\top}(t+\tau)\right\rangle=\delta(\tau)$, and $\varepsilon$ is a noise intensity.

In this paper, we study the effect of random disturbances on the system (2) in the regime of tonic spiking oscillations. For the numerical simulation of random trajectories, the standard Runge-Kutta fourth-order deterministic scheme with corresponding stochastic terms and the time step 0.0001 was used.

Consider the value $I=3.7$. Here, the spiking cycle with one spike per period is the attractor of the deterministic system. Figures 2(a) and 2(b) shows the random trajectories starting from this deterministic cycle (in the projection on $x O z$ plane) and the corresponding $x(t)$ plots for two values of noise intensity. For a sufficiently small noise $(\varepsilon=0.01)$, the random trajectories are localized in some small vicinity of the deterministic limit cycle. The type of oscillations remains spiking, with one spike per 

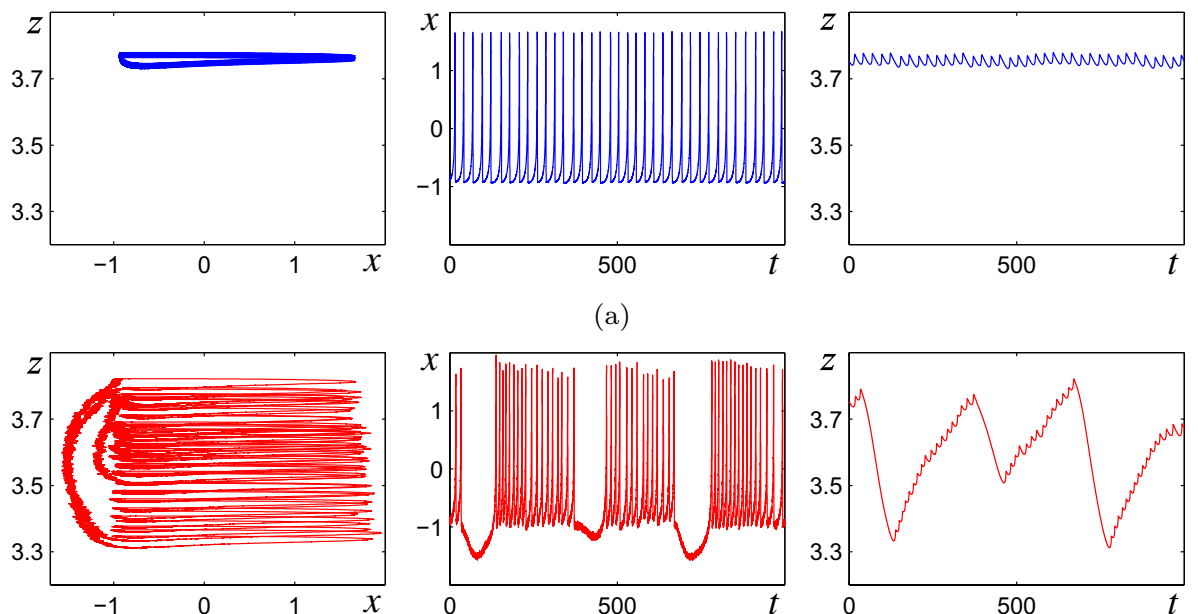

(a)
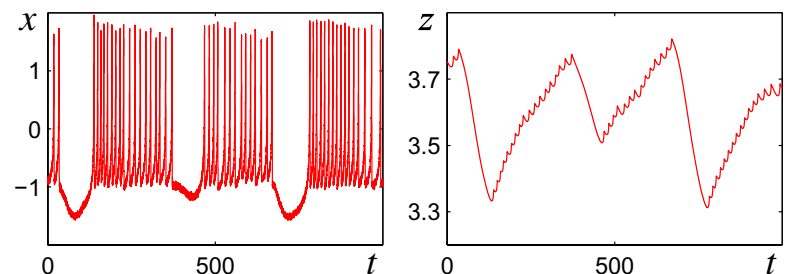

(b)

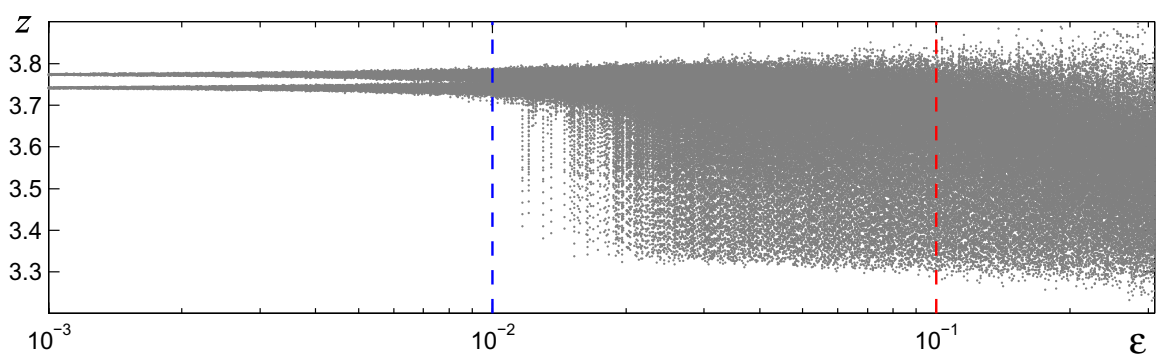

(c)

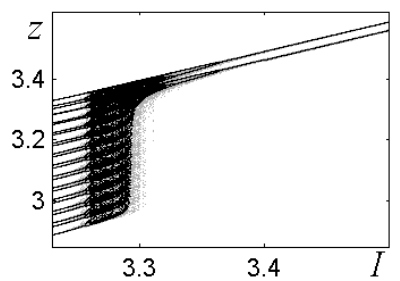

(d)

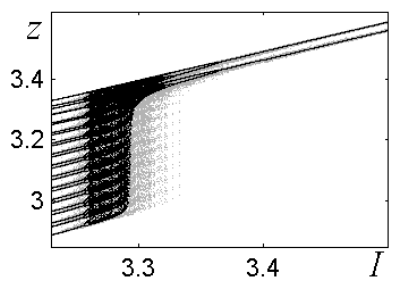

(e)

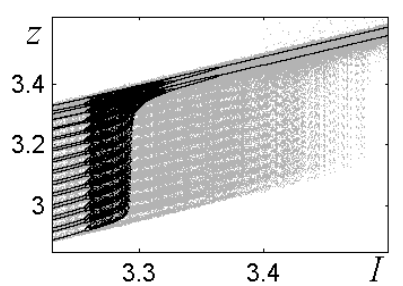

(f)

Fig. 2. Stochastic generation of bursting oscillations for $I=3.7$ : (a) $\varepsilon=0.01$, (b) $\varepsilon=0.1$. For a small noise (a), random trajectories starting from the deterministic limit cycle are localized in a small vicinity of it (noisy spiking regime). With an increase of noise (b), the dynamic regime changes to the stochastic bursting one (the alternation of spiking and quiescent phases and the sharp decrease of $z$-coordinates are observed). (c) Changes in the dispersion of random trajectories for $I=3.7$ : $z$-coordinates of intersection points of random trajectories with Poincare section $x=\bar{x}$ in dependence on the noise intensity. Dashed lines mark the values $\varepsilon=0.01$ and $\varepsilon=0.1$ for which the stochastic dynamics displayed in Figs. 3(d)-3(f) Deterministic (black) and stochastic (gray) bifurcation diagrams: (d) $\varepsilon=0.0005$, (e) $\varepsilon=0.001$, (f) $\varepsilon=0.005$. 
period (Fig. 2(a)). This can be considered as noisy tonic spiking oscillations. If the noise intensity is greater than some threshold value $(\varepsilon=0.1)$, the random trajectories go far from the deterministic cycle, with a sharp decrease of $z$-coordinates, and then an oscillatory transition process of approaching to the deterministic cycle is observed (Fig. 2(b)). On the $x(t)$ plot, one can observe clearly defined intervals of quiescence and spiking phases. This indicates that under noise, the type of oscillations changed from spiking to bursting.

Figure 2(c) shows how noise changes the dispersion of random trajectories for $I=3.7$. Here, $z$-coordinates of points of intersection of random trajectories with the Poincare section plane $x=\bar{x}$ ( $\bar{x}$ is $x$-coordinate of the equilibrium) in dependence of noise intensity are plotted. Dashed lines mark the values $\varepsilon=0.01$ and $\varepsilon=0.1$ for which the stochastic dynamics is displayed in Figs. 2(a) and 2(b). For small noise intensities, random states concentrate near the deterministic limit cycle and have a sufficiently small dispersion. With an increase of noise, a significant deviation of $z$-coordinates in the direction of smaller values is observed. This corresponds to the emergence of bursting oscillations.

Consider how noise changes the bifurcation diagram. Figures $2(\mathrm{~d})-2(\mathrm{f})$ show the stochastic bifurcation diagrams for various values of $\varepsilon$. In the deterministic bifurcation diagram, the transition from the tonic spiking region to the bursting region is characterized by the sharp increase in the range of values of $z$-coordinates (i.e., the expansion of the diagram). Note that in stochastic bifurcation diagrams, the bursting region shifts to the right, i.e., to higher $I$ values (compare the black line with the gray line in Figs. 2(d)-2(f)).

Figure 3 demonstrates the details of the distribution of random trajectories in dependence on the noise intensity. The probability density function $P(z)$ for $z$-coordinates of points of intersection of random trajectories with $x=\bar{x}$ is plotted for $I=3.7$ for various noise intensities. The functions were obtained by averaging the data from 100 realizations of random trajectories on the time interval $[0, T], T=10^{6}$, discarding the transient period $t=10^{5}$.

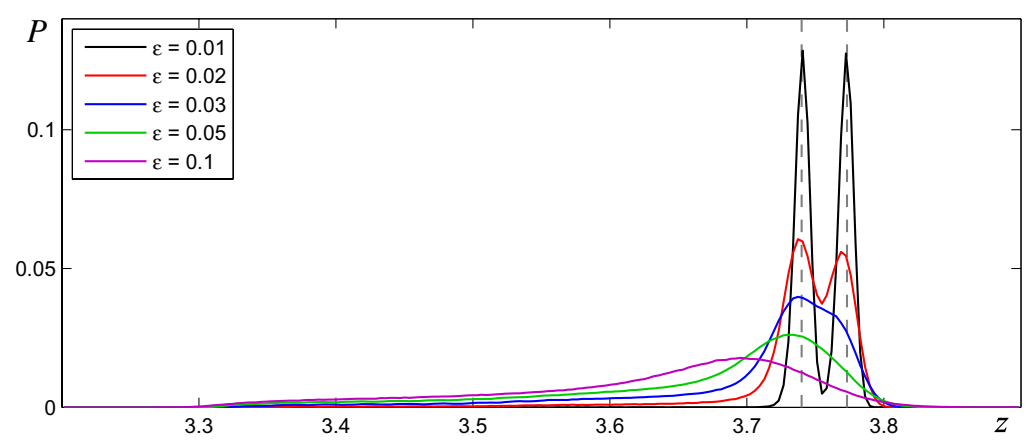

Fig. 3. PDFs for distribution of intersection points of random trajectories with Poincare section $x=\bar{x}$ in dependence on the noise intensity. Dashed lines denote the locations of intersections of the deterministic limit cycle with $x=\bar{x}$. 
For a weak noise $(\varepsilon=0.01)$, the probability density function $P(z)$ is bimodal with the narrow and high peaks located above the corresponding states of the deterministic limit cycle: $z_{1}=3.74$ and $z_{2}=3.77$. The stronger noise $(\varepsilon=0.02)$ slightly smoothens these peaks. The further increase of noise $(\varepsilon=0.05)$ results in the transformation of the form of the function to the unimodal one. For greater noise intensity values, the peak of the function $P(z)$ shifts to the zone with smaller $z$-coordinates.

Let us suggest the criterion for the transition from the spiking regime to the bursting one. Note that for the bursting oscillations, the random states with $x<-1$ are observed (the quiescence zones in Fig. 2(b)). The value $x=-1$ can be used as a threshold that separates in the phase space the quiescence from the spiking phase. For a quantification of the weight of spiking time in the total time of the observation, consider the numerical characteristic $\zeta(\varepsilon)=\lim _{T \rightarrow \infty} \frac{T_{q}}{T}$, where $T_{q}$ is the time spending by the system in the region $x<-1$, and $T$ is the total time. Function $\zeta$ can be considered as the probability for generation of bursts. In Fig. 4(a), functions $\zeta(\varepsilon)$ for different $I$ are plotted. Here, a sufficiently large time of observation $T=10^{7}$ is taken in computations. For a small noise, random states are concentrated near the deterministic cycle, so $\zeta=0$. With the increase of the noise intensity, one can observe a growth of $\zeta$ values. This means that with the increasing noise, the system begins to spend more time in the zone with $x<-1$, which indicates the stochastic generation of bursting.

Stochastic changes in oscillatory dynamics are traditionally quantified by statistics of ISIs $\tau$, such as the probability density function (pdf) of ISIs, the mean ISI $(\langle\tau\rangle)$, and the coefficient of variation $(\mathrm{CV}), C_{V}$, defined as the ratio of the ISIs standard deviation to the mean ISI.

Figure 5 displays the pdfs of ISIs for $I=3.7$ and $I=3.35$ for various noise intensity values. For $I=3.7$, the function has one peak, corresponding to the period of the deterministic cycle $(T=27)$. With the increase of noise $(\varepsilon=0.02)$, a new peak (see the enlarged fragment in Fig. $5(\mathrm{a})$ ) of the function in the zone of long ISIs appears $(\tau \approx 120)$, which corresponds to the emergence of bursting oscillations (a long interval accounts for the quiescence phase). However, the expected increase of the mean ISI does not occur (see Fig. 4(b)). On the contrary, one can observe that the mean ISI decreases with the increase of noise. This effect is due to the fact that simultaneously with the emergence of the long ISIs, the distribution of the short ISIs drastically changes. This can be seen in detail in Fig. 5(a) (left part). One can observe that with the increase of noise $(\varepsilon=0.02)$, the peak of pdf in the zone of short ISIs splits in two peaks, with a new peak corresponding to shorter intervals $(\tau \approx 11)$, which reflects the increase of the frequency of spikes in the emerged burst. With the further increase of noise, the shorter ISIs begin to dominate, and two peaks in the zone of short ISIs merge into one $(\varepsilon=0.1)$. Thus, under random disturbances, the probability density first changes from unimodal to trimodal, and then becomes bimodal. This means that the system undertakes $P$-bifurcation [17] related to the qualitative change of the distribution of ISIs of the stochastic cycle. 


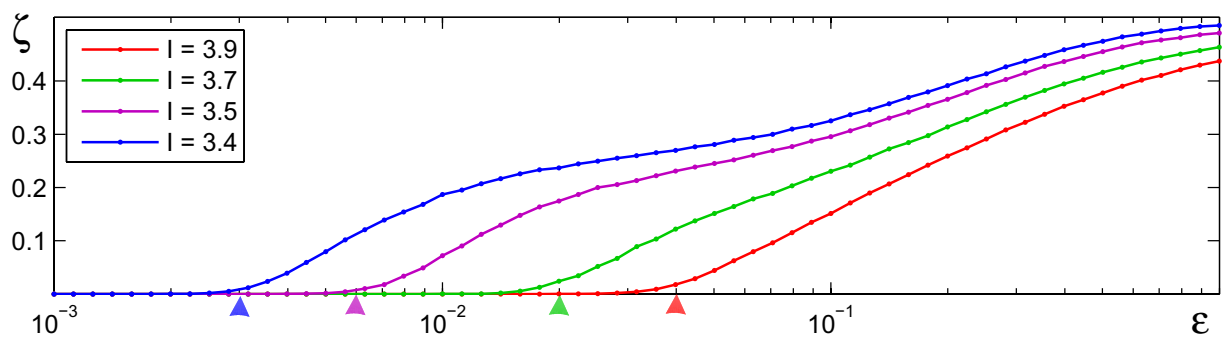

(a)

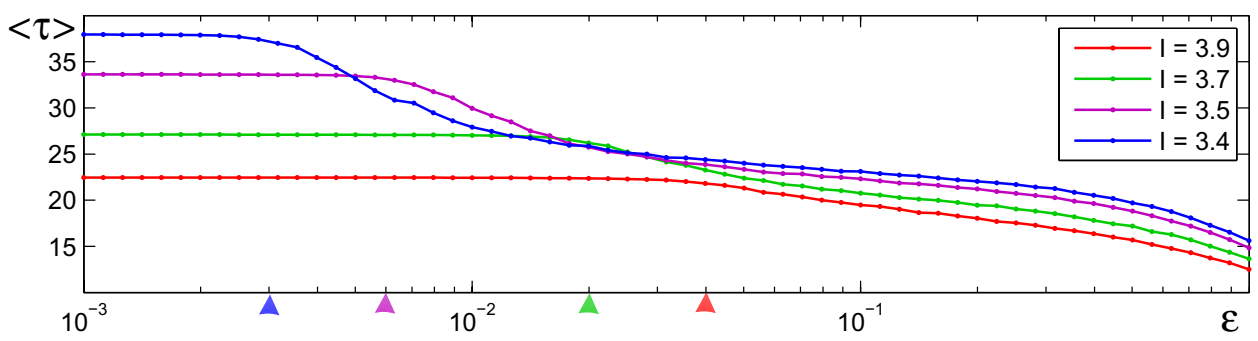

(b)

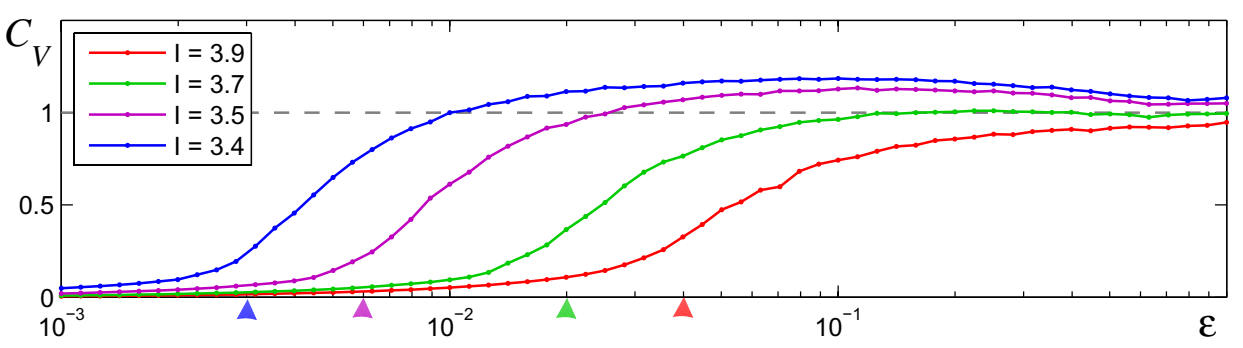

(c)

Fig. 4. (a) Plots of the function $\zeta(\varepsilon)=\lim _{T \rightarrow \infty} \frac{T_{q}}{T}$ in dependence on noise intensity. Here $T_{q}$ is the time spent by the system in the zone $x<-1$ and $T$ is the total time. Here, $T=10^{7}$ is taken for computations. The value $x=-1$ is used as a threshold that separates in the phase space the spiking phase from the quiescent one. A sharp increase of $\zeta$ indicates the onset of the stochastic generation of bursting. The triangles mark the critical values of noise intensity. (b) and (c) ISIs: (b) mean value, (c) coefficient of variation $(\mathrm{CV})$. Dashed line indicates the Poissonian limit $\left(C_{V}=1\right)$. The triangles mark the critical values of noise intensity.

A similar effect is observed for other values of $I$ in the region of spiking cycles. Indeed, for $I=3.35$, the deterministic cycle has two spikes per period. Figure 5(b) shows the pdfs of ISIs for $I=3.35$ for various noise intensity values. Note that for small noise, the pdf is bimodal. With the increase of noise, these peaks merge. With the further increase of noise, as in the case of the cycle with one spike, the new peaks in zones of longer and shorter ISIs appear. 


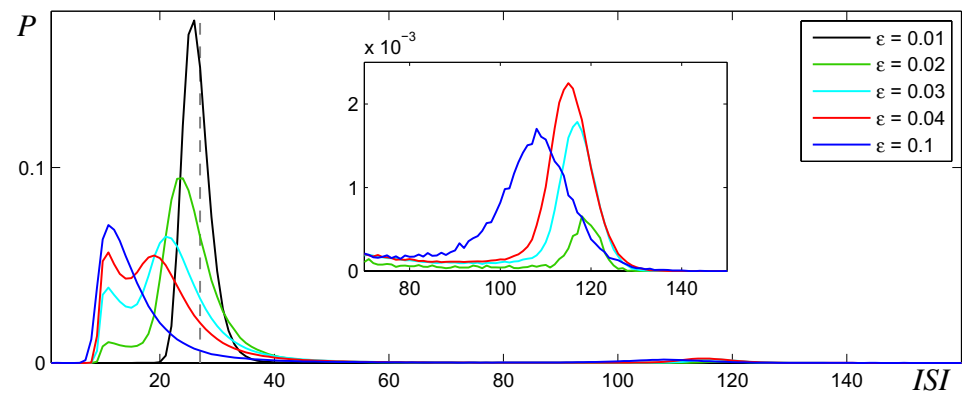

(a)

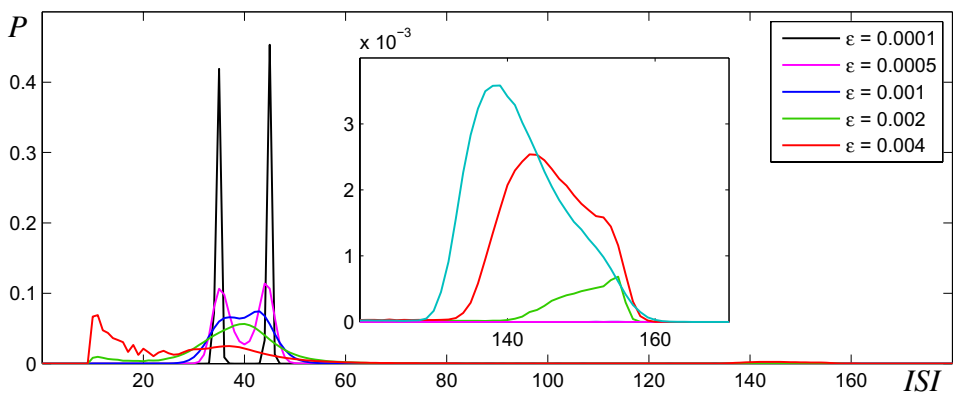

(b)

Fig. 5. PDFs of ISIs for (a) $I=3.7$, (b) $I=3.35$. Dashed lines indicate the values of ISIs for the oscillations in the deterministic system (for $I=3.7$, it is the period of the cycle).

Summarizing, the stochastic transition from spiking to bursting oscillations is characterized by two effects: the emergence of long ISIs, corresponding to the quiescence phase, and the shift of the pdf peaks to the zone of shorter ISIs (i.e., the increase of the frequency of spikes in bursts). Long ISIs are sufficiently rare, therefore the mean ISI decreases with increasing noise intensity.

Figure 4(c) shows the plots of the coefficient of variation (CV) for various $I$ in dependence on the noise intensity $\varepsilon$. For sufficiently small noise intensities, the CV is close to zero. With the increase of noise, one can observe the sharp increase of the $\mathrm{CV}$, which indicates the growth of variability of ISIs under random disturbances. This phenomenon is known as anti-coherence resonance [42] or incoherence resonance [43]. The CV reaches some level, and with the further increase of noise, it stays almost constant and close to the Poissonian limit, i.e., $C_{V}=1$.

Figures 2(c) and 4 allow us to make empirical estimations for the critical values of noise intensity corresponding to the transition from the spiking regime to the bursting one. This transition is accompanied by the several observed effects (the sharp decrease of $z$-coordinates, the emergence of the quiescence zones with $x<-1$, the decrease of the mean ISI, and the growth of the ISI's variance) which appear at some level of noise. For $I=3.4$, we estimate $\varepsilon^{*} \approx 0.003$, for $I=3.5$, we get 
$\varepsilon^{*} \approx 0.006$, for $I=3.7$, we have $\varepsilon^{*} \approx 0.02$, and for $I=3.9$, we get $\varepsilon^{*} \approx 0.04$ (see Fig. 4 , the critical values of noise intensity are marked with triangles).

\section{Stochastic Sensitivity Analysis}

The emergence of noise-induced bursting oscillations can be explained by the peculiarities of the phase portrait of the deterministic system. Trajectories starting close to the deterministic cycle, finally approach the cycle, but the character of the transient process depends on the value of the initial deviation. Indeed, if the deviations are relatively small, the trajectories tend to the cycle monotonically. If we take the initial deviations larger than some threshold, the trajectory goes sufficiently far from the cycle and approaches it making several loops that correspond to spikes (Fig. 6). Thus, considering different initial points, one can specify a border between these two different transient regimes in the phase space. Let us define this border by the term "pseudo-separatrix".

To analyze the mechanism of stochastic generation of bursting oscillations, we apply the SSF technique. SSF allows us to approximate a dispersion of random states around the deterministic attractors. A brief review of the SSF technique is given in the Appendix.

Eigenvalues and eigenvectors of the stochastic sensitivity matrix define a geometric configuration of the confidence domains, i.e., the variance of random states around the stable attractor. Using SSF technique, we can construct a family of confidence ellipses around the stable limit cycle. The eigenvalues of SSF matrix may vary nonuniformly along the limit cycle. This is shown in Figs. 7(a) and 7(b), which displays the nonzero eigenvalues of the SSF matrix of the limit cycle for $I=3.5$ and $I=3.7$. One can observe a significant irregularity of dispersion of random trajectories along the cycle. The largest values of SSF correspond to the spiking

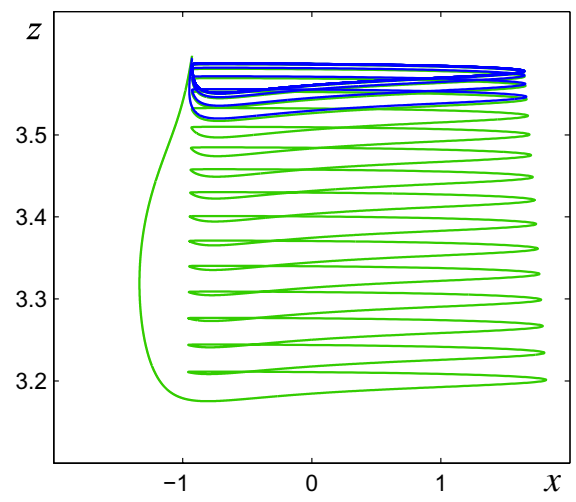

(a)

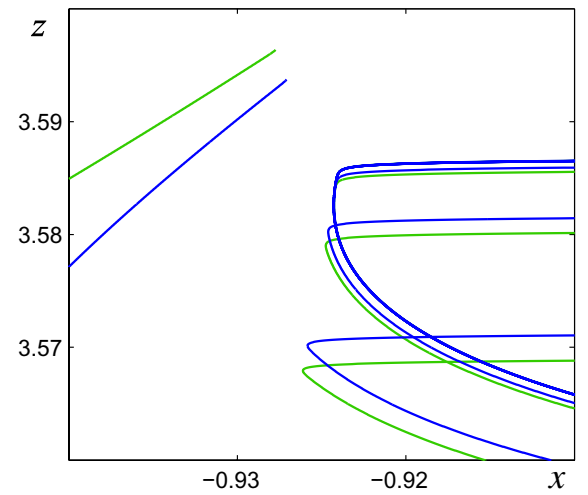

(b)

Fig. 6. Deterministic phase trajectories for $I=3.5$ : the character of transient process depends on initial conditions (here, two colors show the results of different initial values of the model). 


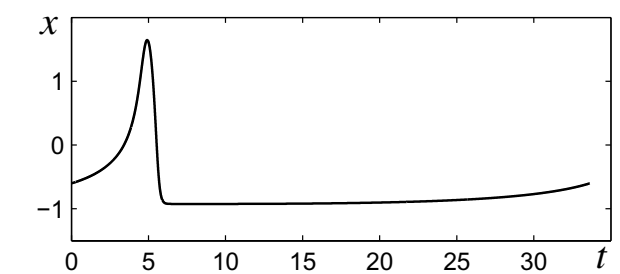

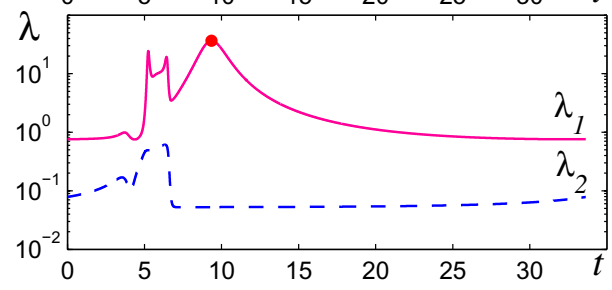

(a)
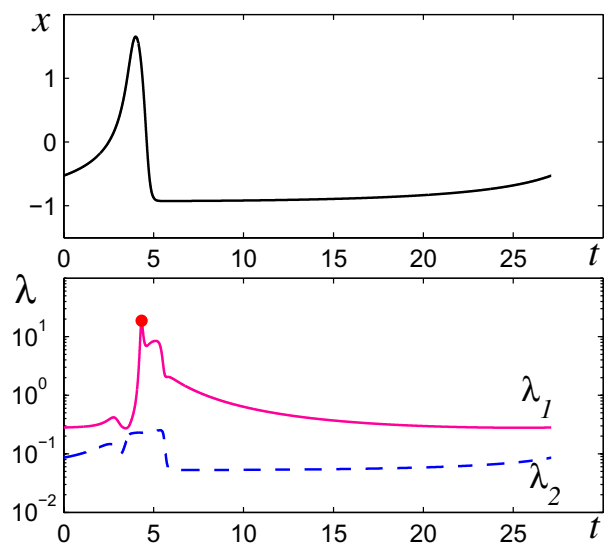

(b)

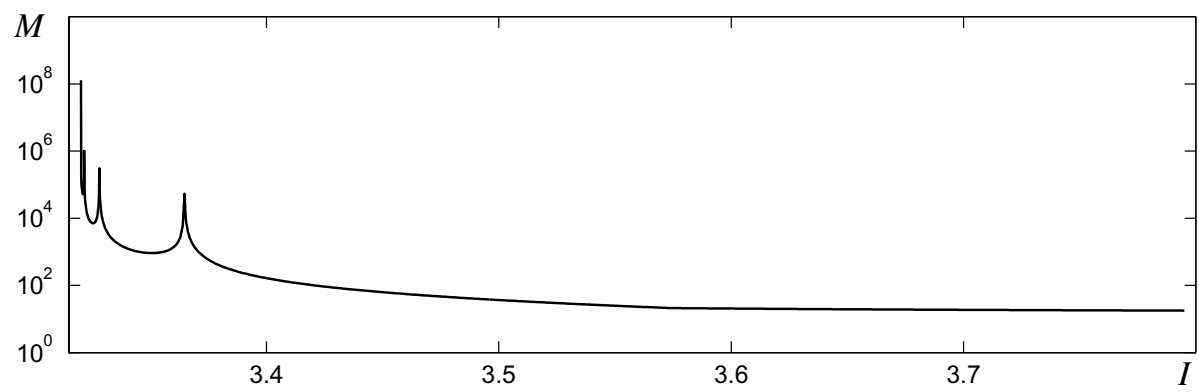

(c)

Fig. 7. Stochastic sensitivity of limit cycles for (a) $I=3.5$, (b) $I=3.7$. The circle marks the location of the stochastic sensitivity factor $M=\max _{[0, T]} \lambda_{1}(t)$. (c) Stochastic sensitivity factor $M$ in the region of tonic spiking cycles.

phase of the limit cycle. The SSF crucially changes under the variation of $I$ : the form of graph for the SSF eigenvalues changes strongly with the variation of $I$, as well as magnitudes of the eigenvalues. Note that these changes of SSF do not result from changes of the phase coordinates of the limit cycle.

To determine the stochastic sensitivity of the cycle as a whole, the stochastic sensitivity factor $M$ can be used (see Appendix). In Fig. 7(c), the plot of the stochastic sensitivity factor $M(I)$ for limit cycles in the spiking region is presented. One can observe that at the points of period-doubling bifurcations, the sensitivity becomes very large. Note that the stochastic sensitivity increases as the period of the cycles grows.

Let us consider a point $\tilde{x}$ of the limit cycle from "transition region", i.e., a part of the cycle from which the stochastic trajectories go off to the bursting zone in the phase space (see Fig. 8). Note that the transition region is not a part of the cycle with maximal stochastic sensitivity, but it is a zone in which large sensitivity combines with the proximity to the pseudo-separatrix. 


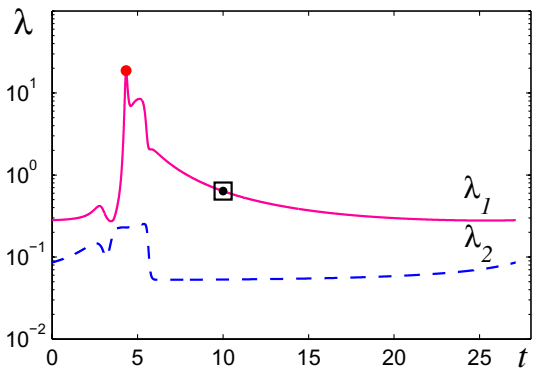

(a)

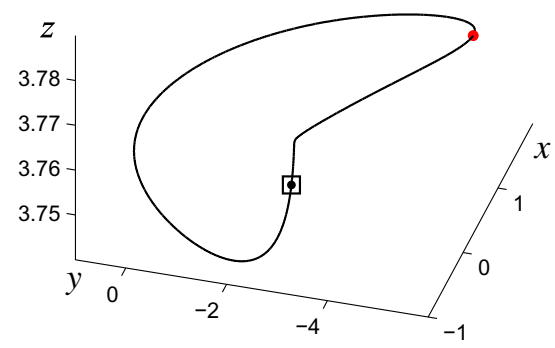

(b)

Fig. 8. Transition region (i.e., a part of the cycle from which the stochastic trajectories go off to the bursting zone) for $I=3.7$ and its stochastic sensitivity: (a) nonzero eigenvalues $\lambda_{1,2}$ of the SSF matrix along the limit cycle; (b) limit cycle in phase space; transition region (square), part of the cycle with highest stochastic sensitivity (red circle).

Consider a hyperplane $\Pi$ that is orthogonal to the cycle at the point $\tilde{x}(t)$. For points of intersection of random trajectories with the plane $\Pi$, we can construct confidence ellipses corresponding to different values of noise intensity. A location, a form and a size of a confidence ellipse are defined by the nonzero eigenvalues $\lambda_{1,2}(t)$ and the corresponding eigenvectors $v_{1,2}(t)$ of the stochastic sensitivity matrix $W(t)$. The equation of the confidence ellipse with the center at the point $\tilde{x}(t)$ in the plane $\Pi$ is the following:

$$
\frac{u_{1}^{2}}{\lambda_{1}}+\frac{u_{2}^{2}}{\lambda_{2}}=2 k^{2} \varepsilon^{2},
$$

where $\left(u_{1}, u_{2}\right)$ are coordinates of the ellipse points in the plane $\Pi$ in the basis $\left(v_{1}, v_{2}\right)$. The parameter $k$ determines a fiducial probability $P=1-e^{-k}$. For a small noise, confidence ellipses give a good approximation for the dispersion of random states around the deterministic cycle (see Fig. 9(a)).

Examining different initial points in the plane $\Pi$, one can define a pseudoseparatrix, specifying a border between two different transient regimes in the phase space. To determine the type of the transient regime (spiking or bursting), we use the threshold value $x=-1$ : if a deterministic trajectory has points with $x<-1$, we assume that the type of the transient regime is bursting.

Let us suggest a method allowing to estimate the critical value of the noise intensity $\varepsilon^{*}$ corresponding to the onset of noise-induced bursting. Figure 9 (b) shows a point of cycle in the transition region for $I=3.7$, pseudo-separatrix, and confidence ellipses for two values of the noise intensity (a fiducial probability is $P=0.99$ ). For the sufficiently small noise intensity $(\varepsilon=0.01)$, the confidence ellipse is close to the deterministic cycle. With an increase of the noise intensity $(\varepsilon=0.02)$, the ellipse expands and intersects the pseudo-separatrix (see Fig. 9(b)). This means that with high probability, stochastic trajectories can go to the bursting zone of the phase 


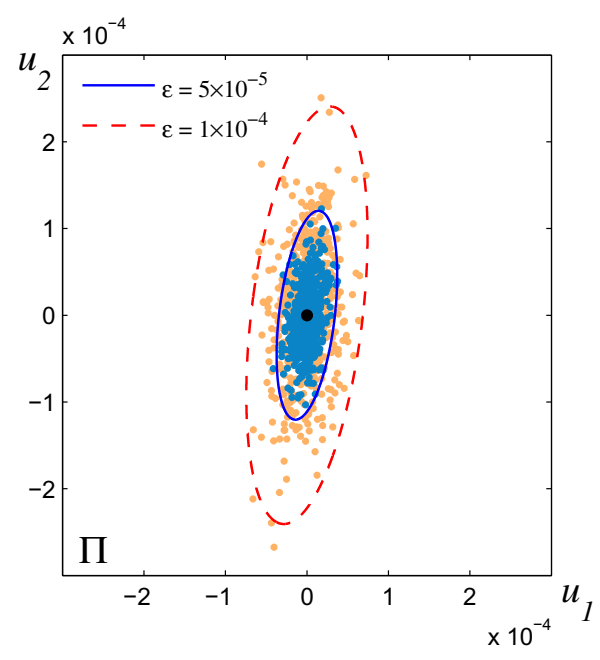

(a)

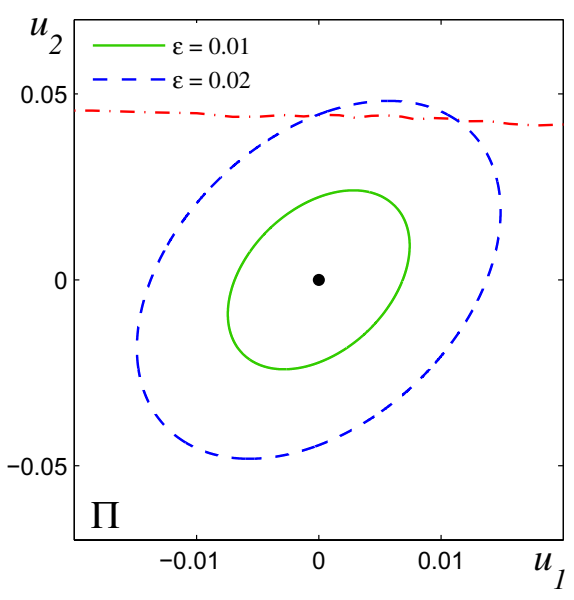

(b)

Fig. 9. Point of deterministic limit cycle (circle) and confidence ellipses (solid, dashed) for $I=3.7$ and the fiducial probability $P=0.99$ with (a) intersection points of random trajectories with normal plane $\Pi$, (b) pseudo-separatrix (dash-dotted).

space. The noise intensity that corresponds to the intersection of the confidence ellipse with the pseudo-separatrix can be used as an estimation of the threshold value $\varepsilon^{*}$. For $I=3.7$, we get $\varepsilon^{*} \approx 0.02$. The obtained value is in a good agreement with the results of the numerical simulations.

Let us describe briefly the procedure of estimation of the critical values for the noise intensity in the following algorithm:

(1) Calculate eigenvalues and eigenvectors of SSF for the limit cycle.

(2) Determine a point $\tilde{x}$ of the limit cycle from "transition region".

(3) Determine a hyperplane $\Pi$ orthogonal to the cycle at the point $\tilde{x}$.

(4) Examining different initial points in the plane $\Pi$, define a pseudo-separatrix.

(5) For the point $\tilde{x}$ and hyperplane $\Pi$, construct a set of confidence ellipses corresponding to different values of noise intensity.

(6) Determine the critical value $\varepsilon^{*}$ of noise intensity as the value for which the confidence ellipse begins to intersect the pseudo-separatrix.

\section{Lyapunov Exponents. Noise-Induced Transitions Between Order and Chaos}

Let us discuss the relations of noise-induced bursting in the system (2) with the behavior of the largest Lyapunov exponent (LLE). The Lyapunov exponent quantitatively reflects the dynamic peculiarities of deterministic and stochastic flows. A negative LLE means that the trajectories of the stochastic system mostly 


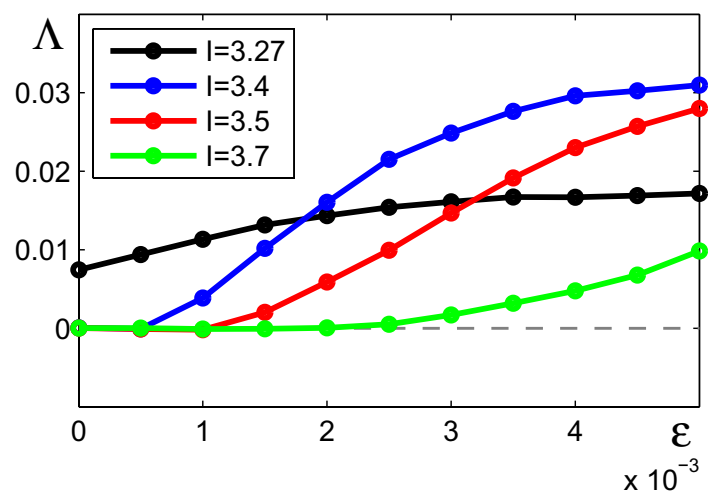

Fig. 10. The largest Lyapunov exponent (LLE) in dependence on the noise intensity. Dashed line denotes $\Lambda=0$.

converge. Positive values of LLE indicate that the divergence dominates. Change of the sign of LLE from negative to positive is widely used as an indicator of the transformation from regular to chaotic dynamics [14-16].

In Fig. 10, Lyapunov exponents $\Lambda(\varepsilon)$ for various $I$ values in dependence on noise intensity are presented. For $I=3.27$, the deterministic system exhibits chaotic bursting, and, correspondingly, for $\varepsilon=0$, Lyapunov exponent is positive. The increase of noise leads to insignificant growth of $\Lambda$.

For values of the parameter $I$ corresponding to the tonic spiking, $\Lambda(0)=0$. With the increase in noise, the values of $\Lambda$ initially decrease slightly, become negative, and then increase sharply and become positive. Note that the values of $\Lambda$ become greater than in the case of the chaotic spiking. Thus, the exponent $\Lambda$ in the region of the tonic spiking is very sensitive to noise. Even for sufficiently small noise intensities, the regular stochastic oscillations transform into the chaotic. Note that the noise-induced chaotization occurs for smaller values of noise intensity than the stochastic generation of bursting oscillations. Thus, the chaotization precedes the generation of bursting.

\section{Summary}

The effect of random disturbances on the HR model with an additive current noise in the parametric region of tonic spiking oscillations was studied. We showed that under noise, the spiking dynamic regime in this model transforms into the bursting one. Along with the changes of the distribution of random trajectories in the phase space, the transformation of the pdf for a distribution of ISIs is observed. It was found that the stochastic transition from spiking to bursting in the HR model is characterized by two effects: the emergence of long ISIs, corresponding to the appearance of quiescence phase, and the increase of the frequency in generated bursts. For a quantitative analysis of the noise-induced bursting, we suggested a 
constructive approach based on the stochastic sensitivity function technique and the method of confidence domains that allows us to describe geometrically a distribution of random states around the deterministic attractors. Using this approach, we developed a new algorithm for the estimation of critical values for the noise intensity corresponding to the qualitative changes in stochastic dynamics. We showed that the obtained values are in a good agreement with direct numerical simulations. A relationship between noise-induced bursting and transitions from order to chaos was discussed, and it was shown that in the HR model, the noise-induced chaotization precedes the stochastic generation of bursts.

\section{Appendix A. Stochastic Sensitivity Function Technique}

Consider a general nonlinear system of Ito stochastic differential equations:

$$
\dot{x}=f(x)+\varepsilon \sigma(x) \xi(t) .
$$

Here, $x$ is an $n$-vector, $f(x)$ is a smooth $n$-dimensional function, $\xi(t)$ is an $n$-dimensional white Gaussian noise with $\left\langle\xi(t) \xi^{\top}(t+\tau)\right\rangle=\delta(\tau) I, I$ is an $n \times n$ identity matrix, $\sigma(x)$ is an $n \times n$ matrix function, and $\varepsilon$ is a scalar parameter of noise intensity.

Let the corresponding deterministic system $(\varepsilon=0)$ have an exponentially stable limit cycle $\Gamma$, defined by a $T$-periodic solution $\bar{x}(t)=\bar{x}(t+T)$.

Trajectories of the randomly forced system (A.1) leave the deterministic cycle $\Gamma$ and form some probabilistic distribution around it. Time evolution of this distribution is governed by FP equation. In a steady regime, one can consider stationary $\rho(x, \varepsilon)$ described by the stationary $\mathrm{FP}$ equation.

A direct usage of this equation is complicated for multidimensional $(n \geq 2)$ systems, therefore various approximations and asymptotics are developed [18, 44, 45]. Here, a well-known quasipotential method [24, 25] and a SSF technique [26, 27, 46] can be applied.

Let $\Pi_{t}$ be a hyperplane that is orthogonal to the cycle at the point $\bar{x}(t)$ $(0 \leq t<T)$. For this plane, in the neighborhood of the point $\bar{x}(t)$, a Gaussian approximation of the stationary probabilistic distribution can be written [26] as:

$$
\rho_{t}(x, \varepsilon)=K \exp \left(-\frac{(x-\bar{x}(t))^{\top} W^{+}(t)(x-\bar{x}(t))}{2 \varepsilon^{2}}\right),
$$

with the mean value $m_{t}=\bar{x}(t)$ and the covariance matrix $D(t, \varepsilon)=\varepsilon^{2} W(t)$. Here, the matrix function $W(t)$ is singular, and the sign "+" means a pseudoinversion. The matrix $W(t)$ is a unique solution of the boundary problem

$$
\dot{W}=F(t) W+W F^{\top}(t)+P(t) S(t) P(t),
$$

with conditions

$$
W(T)=W(0), \quad W(t) f(\bar{x}(t))=0 .
$$


Here

$$
\begin{aligned}
F(t) & =\frac{\partial f}{\partial x}(\bar{x}(t)), \quad S(t)=\sigma(\bar{x}(t)) \sigma^{\top}(\bar{x}(t)), \\
P(t) & =I-\frac{f(\bar{x}(t)) f^{\top}(\bar{x}(t))}{f^{\top}(\bar{x}(t)) f(\bar{x}(t))} .
\end{aligned}
$$

The matrix $W(t)$ characterizes the stochastic sensitivity of the system to random disturbances. We will term it stochastic sensitivity function (SSF) matrix. The eigenvalues $\lambda_{i}(t)$ and the eigenvectors $v_{i}(t)$ of the SSF matrix describe the dispersion of random states in the Poincare section $\Pi_{t}$ near the point $\bar{x}(t)$ of the cycle.

The eigenvalues and eigenvectors of the SSF matrix can be found via the method of singular decomposition described in [26]. This approach was effectively applied for the analysis of the three-dimensional stochastic systems in $[47,48]$.

The value $M=\max _{[0, T]} \lambda_{1}(t)$ is a useful characteristic of the cycle as a whole. We consider $M$ as a stochastic sensitivity factor of the limit cycle $\Gamma$.

SSF matrix allows to construct a confidence ellipse with the center in the point $\bar{x}(t)$. The equation of this ellipse in the plane $\Pi_{t}$ looks like

$$
(x-\bar{x}(t))^{\top} W^{+}(t)(x-\bar{x}(t))=2 k^{2} \varepsilon^{2},
$$

where the parameter $k$ determines a fiducial probability $P=1-e^{-k}$. A set of these ellipses for $t \in[0 ; T)$ specify some confidence torus around a deterministic cycle. This torus is a confidence domain in a phase space for the stochastic cycle as a whole [47].

\section{Acknowledgments}

The work was supported by Russian Science Foundation (N 16-11-10098).

\section{References}

[1] E. M. Izhikevich, Dynamical Systems in Neuroscience: The Geometry of Excitability and Bursting (MIT Press, Cambridge, 2007).

[2] R. R. Llinas, Intrinsic electrical properties of mammalian neurons and CNS function: A historical perspective, Front. Cell. Neurosci. 8 (2014) 320.

[3] A. L. Hodgkin and A. F. Huxley, A quantitative description of membrane current and its application to conduction and excitation in nerve, J. Physiol. 117 (1952) 500-544.

[4] R. FitzHugh, Impulses and physiological states in theoretical models of nerve membrane, Biophys. J. 1 (1961) 445-466.

[5] B. Lindner, J. Garcia-Ojalvo, A. Neiman and L. Schimansky-Geier, Effects of noise in excitable systems, Physics Rep. 392 (2004) 321-424.

[6] C. Morris and H. Lecar, Voltage oscillations in the barnacle giant muscle fiber, Biophys. J. 35 (1981) 193-213.

[7] K. Tsumoto, H. Kitajima, T. Yoshinaga, K. Aihara and H. Kawakami, Bifurcations in Morris-Lecar neuron model, J. Neurocomput. 69 (2006) 293-316.

[8] J. L. Hindmarsh and R. M. Rose, A model of neuronal bursting using three coupled first order differential equations, Proc. R. Soc. Lond. B Biol. Sci. 221 (1984) 87-102.

[9] A. Destexhe and M. Rudolph-Lilith, Neuronal Noise (Springer, New-York, 2012). 
[10] V. S. Anishchenko, V. V. Astakhov, A. B. Neiman, T. E. Vadivasova and L. SchimanskyGeier, Nonlinear Dynamics of Chaotic and Stochastic Systems. Tutorial and Modern Development (Springer-Verlag, Berlin, 2007).

[11] L. Gammaitoni, P. Hanggi, P. Jung and F. Marchesoni, Stochastic resonance, Rev. Mod. Phys. 70 (1998) 223-287.

[12] M. D. McDonnell, N. G. Stocks, C. E. M. Pearce and D. Abbott, Stochastic Resonance: From Suprathreshold Stochastic Resonance to Stochastic Signal Quantization (Cambridge University Press, Cambridge, 2008).

[13] W. Horsthemke and R. Lefever, Noise-Induced Transitions (Springer, Berlin, 1984).

[14] J. B. Gao, S. K. Hwang and J. M. Liu, When can noise induce chaos? Phys. Rev. Lett. 82 (1999) 1132-1135.

[15] F. Gassmann, Noise-induced chaos-order transitions, Phys. Rev. E 55 (1997) 2215-2221.

[16] K. Matsumoto and I. Tsuda, Noise-induced order, J. Stat. Phys. 31 (1983) 87-106.

[17] L. Arnold, Random Dynamical Systems (Springer-Verlag, Berlin, 1998).

[18] B. Lindner and L. Schimansky-Geier, Analytical approach to the stochastic FitzHughNagumo system and coherence resonance, Phys. Rev. E 60 (1999) 7270-7276.

[19] A. S. Pikovsky and J. Kurths, Coherence resonance in a noise-driven excitable system, Phys. Rev. Lett. 78 (1997) 775-778.

[20] P. E. Kloeden and E. Platen, Numerical Solution of Stochastic Differential Equations (Springer-Verlag, Berlin, 1992).

[21] R. Mannella, A gentle introduction to the integration of stochastic differential equations, Stochastic Processes in Physics, Chemistry, and Biology, Vol. 55 (Berlin, Heidelberg: Springer, 2000), pp. 353-364.

[22] R. Mannella, Integration of stochastic differential equations on a computer, Int. J. Mod. Phys. C 13 (2002) 1177-1194.

[23] H. Risken, The Fokker-Planck Equation. Methods of Solution and Applications (Springer-Verlag, Berlin, 1984).

[24] M. Dembo and O. Zeitouni, Large Deviations Techniques and Applications (Jones and Bartlett Publishers, Boston, 1995).

[25] M. I. Freidlin and A. D. Wentzell, Random Perturbations of Dynamical Systems (Springer, New York, 1984).

[26] I. A. Bashkirtseva and L. B. Ryashko, Stochastic sensitivity of 3D-cycles, Math. Comput. Simul. 66 (2004) 55-67.

[27] I. Bashkirtseva and L. Ryashko, Sensitivity and chaos control for the forced nonlinear oscillations, Chaos Solitons Fractals 26 (2005) 1437-1451.

[28] X.-J. Wang, Genesis of bursting oscillations in the Hindmarsh-Rose model and homoclinicity to a chaotic saddle, Physica D 63 (1993) 263-274.

[29] G. Innocenti, A. Morelli, R. Genesio and A. Torcini, Dynamical phases of the Hindmarsh-Rose neuronal model: Studies of the transition from bursting to spiking chaos, Chaos 17 (2007) 043128.

[30] A. Shilnikov and M. Kolomiets, Methods of the qualitative theory for the HindmarshRose model: A case study -a tutorial, Int. J. Bifurcation Chaos 18 (2008) 2141-2168.

[31] M. Desroches, T. Kaper and M. Krupa, Mixed-mode bursting oscillations: Dynamics created by a slow passage through spike-adding canard explosion in a square-wave burster, Chaos 23 (2013) 046106.

[32] R. Barrio, M. A. Martinez, S. Serrano and A. Shilnikov, Macro- and micro-chaotic structures in the Hindmarsh-Rose model of bursting neurons, Chaos 24 (2014) 023128.

[33] R. Barrio, M. Lefranc, M. A. Martinez and S. Serrano, Symbolic dynamical unfolding of spike-adding bifurcations in chaotic neuron models, Europhys. Lett. 109 (2015) 20002. 
[34] A. Longtin, Autonomous stochastic resonance in bursting neurons, Phys. Rev. E 55 (1997) 868-876.

[35] V. V. Osipov and E. V. Ponizovskaya, Multivalued stochastic resonance in a model of an excitable neuron, Phys. Lett. A 271 (2000) 191-197.

[36] J. Baltanas and J. Casado, Noise-induced resonances in the Hindmarsh-Rose neuronal model, Phys. Rev. E 65 (2002) 041915.

[37] S. Reinker, E. Puil and R. M. Miura, Resonances and noise in a stochastic HindmarshRose model of thalamic neurons, Bull. Math. Biol. 65 (2003) 641-663.

[38] H. Gu, M. Yang, L. Li, Z. Liu and W. Ren, Experimental observation of the stochastic bursting caused by coherence resonance in a neural pacemaker, Neuroreport 13 (2002) $1657-1660$.

[39] S. Xia and L. Qi-Shao, Coherence resonance and synchronization of Hindmarsh-Rose neurons with noise, Chin. Phys. 14 (2005) 1088-1094.

[40] I. Bashkirtseva, L. Ryashko and E. Slepukhina, Order and chaos in the stochastic Hindmarsh-Rose model of the neuron bursting, Nonlinear Dyn. 82 (2015) 919-932.

[41] J. Rinzel, A formal classification of bursting mechanisms in excitable systems, in Proc. Int. Congress Mathematicians (Providence, RI: American Mathematical Society, 1987), pp. 1578-1594.

[42] A. M. Lacasta, F. Sagues and J. M. Sancho, Coherence and anticoherence resonance tuned by noise, Phys. Rev. E 66 (2002) 045105.

[43] B. Lindner, L. Schimansky-Geier and A. Longtin, Maximizing spike train coherence or incoherence in the leaky integrate-and-fire model, Phys. Rev. E 66 (2002) 031916.

[44] C. Kurrer and K. Schulten, Effect of noise and perturbations on limit cycle systems, Physica D 50 (1991) 311-320.

[45] G. N. Mil'shtein and L. B. Ryashko, A first approximation of the quasipotential in problems of the stability of systems with random non-degenerate perturbations, J. Appl. Math. Mech. 59 (1995) 47-56.

[46] I. Bashkirtseva, L. Ryashko and E. Slepukhina, Noise-induced oscillation bistability and transition to chaos in FitzHugh-Nagumo model, Fluct. Noise Lett. 13 (2014) 1450004.

[47] L. Ryashko, I. Bashkirtseva, A. Gubkin and P. Stikhin, Confidence tori in the analysis of stochastic 3D-cycles, Math. Comput. Simul. 80 (2009) 256-269.

[48] L. Ryashko, I. Bashkirtseva and P. Stikhin, Noise-induced chaos and backward stochastic bifurcations in the Lorenz model, Int. J. Bifurcation Chaos 23 (2013) 1350092. 